\title{
Customized configuration for hierarchical products: component clustering and optimization with PSO
}

\author{
Yiliu Liu • Zhenyou Zhang • Zixian Liu
}

Received: 12 October 2010 / Accepted: 20 February 2011 / Published online: 10 May 2011

(C) The Author(s) 2011. This article is published with open access at Springerlink.com

\begin{abstract}
Most of the current studies on product configuration suppose modules in configuration are well-defined. In fact, if a component is regarded as the basic configuration item in a hierarchical product, it is worth studying how to cluster components in a better way to form modules satisfying some requirements for product diversity and cost. The authors identify the possible computation scale of a component clustering issue, represent it with network diagram, and apply discrete particle swarm optimization (PSO) to seek a desired clustering way of components. A mobile printer is introduced to exemplify the calculation procedure of the proposed method, and some components of the product are decomposed further so to evaluate the adaptability of PSO to the change of computation scale. The effectiveness of the method is validated in a wide range.
\end{abstract}

Keywords Product configuration - Component clustering $\cdot$ Large-scale optimization •

Particle swarm optimization

Manufacturing companies are offering more product variety and diversity as a response to heterogeneous customer needs $[1,2]$. The goal of design shifts from designing individual products toward designing families

\footnotetext{
Y. Liu $\cdot$ Z. Zhang $(\varangle)$

Department of Production and Quality Engineering, NTNU, Trondheim, Norway

e-mail: zhenyou.zhang@ntnu.no

Y. Liu · Z. Liu

School of Management, Tianjin University, Tianjin, China
}

of products [3, 4], so to provide a set of differently specific products. Generally, varying requirements of customers may lead to some difference in red heir finally selected product models. For a certain requirement, there should be a desired option, on the basis of which the engineers can continue to perform detailed design [5]. The desired solution determination process for a certain requirement is called product configuration.

Product configuration helps manufacturers through generating a desired bill of materials (BOM) to provide customers with satisfied products based on a product family, and it has been applied in diverse industry fields. Evaluations on configuration and selection of schemes are attractive research topics in the past decade. However, a supposition of existing studies is that a product in configuration has been well partitioned, namely its components have been clustered in the best way. Here, to partition a product means to group/cluster all components in a product into several modules as units in configuration. Researches subconsciously assume modules, the component groups in configuration, as fixed, and the grouping way as most appropriate for the product, if not the only one. In fact, before the configuration system appears in front of customers, it needs to be validated that the component clustering approach is optimal. The objective of this article is to propose a method to justify the optimized partition of the product model for customer oriented configuration.

The remainder of this paper is organized as follows: Related literature is reviewed in Section 2, and then the authors discuss the issues in clustering components and illustrate its possible computation scale. An optimization method based on particle swarm optimization (PSO) is proposed, and its effectiveness is discussed 
with a practical example. Conclusions appear at the end.

\section{Related works}

In terms of product configuration, other two concepts should not be ignored: product architecture and modularity. Literature [6] defined product architecture as the arrangement of functional elements, the mapping from functional elements to physical components, and the specification of the interfaces among interacting physical components. Given the subsystem as an intermediate level between the component and product levels, from a network perspective, product architecture can be characterized by representing subsystems as nodes and interfaces between subsystems as links [6,7].

Subsystems are always called modules in the studies on modularity. Modularity means to divide a final product model into smaller units and the interconnections between groups of components, so to create a variety of products with common and independent parts [8-10]. Modular design use interchangeable and configurable modules with "mix-and-match capability" [9]. A module in such kind of design is a collection of components that demonstrates the maximum commonalty and similarity in product structure according to the constraints of product variety, cost, customer requirement, etc. [11]. In the paper of [12], the authors highlighted the attributes of a basic module in modular product design: substantiality, independence, functionality, and relevance.

Product configuration focuses on specifying physical components for some requirements on the basis of modular design. The definition of configuration is firstly given by [13], where a component is expressed with a set of properties; configuration is a set of components and a description of the connections between the components in the set. Then, product configuration is gradually regarded as a generative procedure choosing suitable and compatible components for a product (generally one component is selected for each type), arranging them and then seeking optimal options [3, $14,15]$. Tasks of configuration include selecting components, associating, arranging, establishing the relationships between the components, and evaluating that concerns the compatibility of selection and goal satisfaction [16] .

Product configuration significantly contributes to increase the operational effectiveness and efficiency when a company translates the customer's needs into product documentation [17]. Since product configuration is the relative spatial and logical arrange- ment of the different parts [18], graphic representations have been widely accepted. Based on the product family architecture (PFA) model proposed by [4], product configuration network models have been constructed $[15,19]$. It can describe compatibility and coupling strength between different components and generate new product variants according to requirements. Furthermore, a configuration task is always restricted as a constraint-satisfaction problem [5, 14], and product configuration is regarded as a constraint optimization process [19-21].

Optimization variables in these researches are always modules or subsystems, and the process of optimization includes differentiating properties of modules with same functions and then comparing the final performance of product models combined by modules with same functions but different parameters. Modules are regarded as "enclosures," where types of involved components are stable, as well as their functions. The comparison is based on the only difference in parameters of components. However, if the component level is regarded as the basis of configuration and a module is viewed as an assembly of components, the module can be taken as open and flexible rather than enclosed and stable. It can be expected to cluster components and create modules for configuration according to a certain requirement. Here, the clustering procedure can be viewed as the pre-configuration if compared with traditional product configuration, and the desired clustering way would guarantee the output of traditional configuration. Similar with optimal solution of configuration, the desired clustering way of components is also based on customers' requirements and the profit target of the manufacturer. In this paper, the authors hope to develop a method to customize modules in a product family. Following issues should be addressed in the next sections:

- How to define and represent a clustering way of components

- How to compare different clustering ways of components and determine a desired one to satisfy some requirement

- How to tackle with the hierarchical structure in consideration of components, modules, and products in a product architecture

- How to find an optimal one in feasible time when there are too many selectable clustering ways

Interfaces and between components are assumed as standard in this study, so the problems about constraints and compatibility are ignored. Besides, the solution space in configuration is supposed as stable, 
which means no new component is introduced during the process of configuration.

\section{Clustering of components}

\subsection{Clustering}

The basic analysis item in this study is a component. According to the definition given by [13], a component in configuration is a set of properties, ports for connection to other components, as well as connection constraints and structural constraints. Here, constraints in connection and structure are ignored, and any physical part fulfilling a necessary function can be regarded as a component and can be viewed a variable in configuration.

Clustering means grouping a number of similar things. Its definition in this paper is grouping components so to create modules for a product family. In other words, to cluster components can be understood as to partition a product into several parts/modules at the component level.
The network approach used by [22] in defining measures of component modularity is also accepted in this paper. In representation, as shown in Fig. 1, the authors use a circle to denote a component, shading area as a module clustered by one or more components, and a line as the connection between two components. Connections do not necessarily exist between each two components due to functional and spacial constraints. Each part in Fig. 1 is a clustering way, which refers to a component clustering/grouping approach.

\subsection{Hierarchical structure}

Current researches on product configuration are based on a two-level product model, and all units in configurations are at the same level. Actually, many products have hierarchical structures, and some modules in product configuration can be broken down further. Take the motherboard of a personal computer (PC) as an example. It is always regarded as a unit in PC configuration standing on the side of common customers; however, it possesses several "sub-modules" as well, such as BIOS chip set, I/O ports, controlling chip,
Fig. 1 Possible clustering ways for two, three, and four components
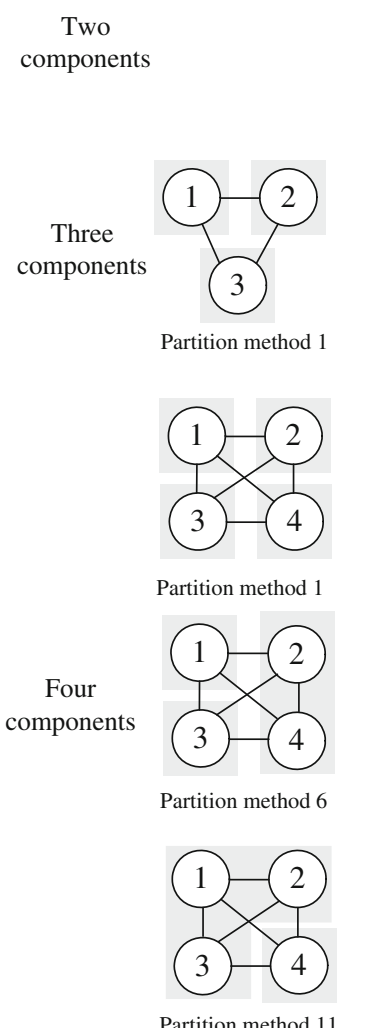

Partition method 11
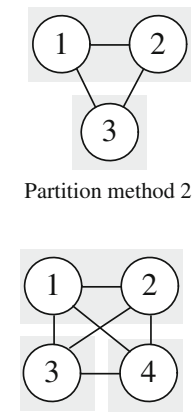

Partition method 2
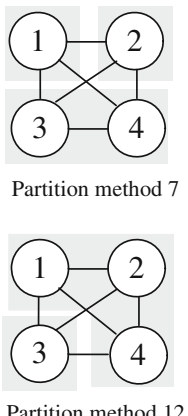

Partition method 12
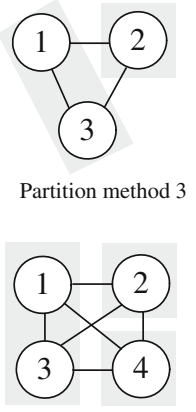

Partition method 3

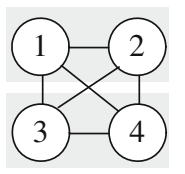

Partition method 8

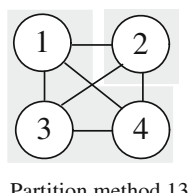

Partition method 13
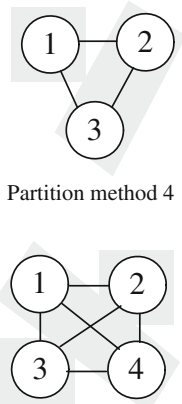

Partition method 4

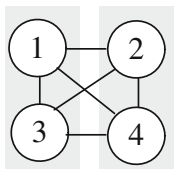

Partition method 9

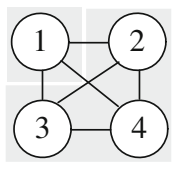

Partition method 14
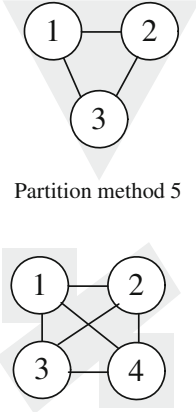

Partition method 5

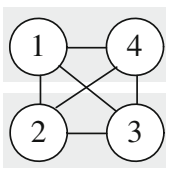

Partition method 10

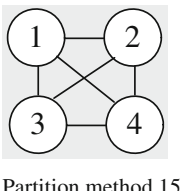

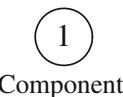

Module

Connection 
audio card, network card, etc. Such sub-modules also can be customized according to certain requirements, e.g., a customer can select a wireless LAN card with WiMAX or not.

In addition, configuration starts from a PFA model in this study. Such an architecture is represented as a tree model [4]. In the present paper, the authors regard a decomposable module as a top of a branch in the whole tree model and its sub-modules as leafs/nodes of the branch. In fact, the branch is also a complete tree if it is placed separately. Thus, the clustering method for the whole model is suitable for any branch of the tree.

On the other hand, some independent modules can be integrated into another module as one of its parts. For example, the graphic card can be regarded as a separate module in configuration or as a part of the motherboard. In this study, given module $A$ can be integrated into module $B$, if there is another module $C$ which connects $A$ but does not connect $B, A$ is independent variable from $B$ in clustering process; otherwise, $A$ is regarded as a part of $B$.

\subsection{Computation scale}

When a product only contains one component, there is only one clustering method; when two components exist, two partition methods are applicable as shown in Fig. 1. Then, with the increase of components, the number of partition ways rises exponentially. Figure 1 shows different partition ways for products with three and four components.

To determine the computing scale, the authors consider two extreme cases: the case where each component only connect one of other components and the case where every component can connect all the others. These two cases are the simplest and the most complex, respectively, for configuration. Given a product with $n$ components, in the former case, there are $n-1$ connections among components, and in the latter case, the number of connection is $C_{n}^{2}$. In this study, the authors partition a product through controlling connections. The state of a connection between two components can be connected or disconnected, describing whether these two components are in the same module or not. Thus, in calculation, the valuing of a connection is binary with 1 or 0 . The possible number $N$ of clustering ways for $n$ components in a product is in the range between the minimum and the maximum following the equation:

$2^{n-1}<N<2^{C_{n}^{2}}$

From the equation, if the amount of components in a product is huge, although some components cannot be combined with others into a module due to cer- tain engineering constraints, numerous computations are unavoidable to approach the optimization. For the hierarchical cases, the amount of clustering ways of components in each branch can be calculated based on the above equation. The total amount of clustering ways is the multiple of numbers of all branches and the root part of the tree. Thus, if there are several branches in a product family architecture, possible clustering ways are more considerable.

\section{Optimization method}

\subsection{Objective function}

The objective of optimization is to find a desired clustering way in considering the kinds of requirements. Due to the existence of varied requirements, the final objective function should be the combination of multiple objectives. Such a final objective and sub-objectives can be set according to practical requirements and constraints.

For each clustering way of components, a certain product varieties can be offered. The number of variety with a clustering way definitely is a measure in evaluation. Besides, compared with optimization among different configuration schemes, the calculation in clustering ways should be based on averages of relevant values of all schemes that can be configured in this clustering way.

\subsection{Particle swarm optimization}

To tackle with the challenges from the scale of calculation, it is necessary to introduce an artificial intelligence method to seek the optimal in the large solution space. Here, the authors choose PSO as the optimization approach for its adaptability and quick converging capacity.

No former relevant research models product configuration as a large-scale optimizing issue for component grouping approaches, and so it is unknown which optimization method is more appropriate. PSO is selected partly because it has been used in solving similar problems such as part machine grouping [23] and manufacturing cell design [24], as well as it is found more robust [25]. On the other hand, the authors prefer PSO for its high efficiency in maintaining the diversity of the swarm, ease to adjust parameters, and no requirement for differentiable optimization problem. 


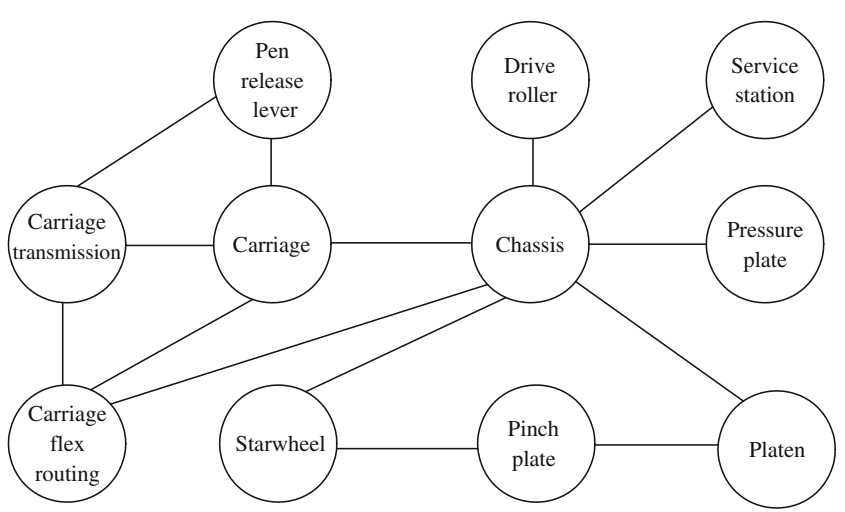

Fig. 2 Configuration structure of a mobile printer

\subsubsection{Description and procedure of PSO}

The initial ideas on particle swarms by Kennedy and Eberhart [26, 27] were essentially aimed at producing computational intelligence by exploiting simple analogues of social interaction. These thoughts soon developed into a powerful optimization methodPSO $[26,28]$, which has been proved very effective for solving global optimization in various engineering applications.

PSO algorithm is a heuristic approach motivated by the observation of social behavior of composed organisms such as birds flocking. A number of simple entities-the particles-are placed in the search space of some problem or function, and each evaluates the objective function at its current location. Each individual in the particle swarm is composed of $D$-dimensional vectors, where $D$ is the dimensionality of the search space. These are the current position $\vec{x}_{i}$, the previous best position $\overrightarrow{p_{g}}$, and the velocity $\vec{v}_{i}$.

The current position $\overrightarrow{x_{i}}$ can be considered as a set of coordinates describing a point in space. If current position is better than any that has been found so far, then the coordinates are stored in the vector $\vec{p}_{i}$. The value of the best function result so far is stored in a

Table 1 Costs of components in the printer

\begin{tabular}{lc}
\hline Component & Cost $(\$)$ \\
\hline Carriage & 20 \\
Carriage & 5 \\
$\quad$ transmission & \\
Chassis & 30 \\
Drive roller & 4 \\
Flex routing & 8 \\
Pen release lever & 8 \\
Pinch plate & 4 \\
Platen & 8 \\
Pressure plate & 6 \\
Service station & 10 \\
Starwheel & 5 \\
\hline
\end{tabular}

variable that can be called $\vec{p}_{g}$ (for "previous best"). The objective, of course, is to keep finding better positions and updating $\vec{p}_{i}$ and $\vec{p}_{g}$. New points are chosen by adding $\overrightarrow{v_{i}}$ coordinates to $\overrightarrow{x_{i}}$, and the algorithm operates by adjusting $\overrightarrow{v_{i}}$, which can effectively be seen as

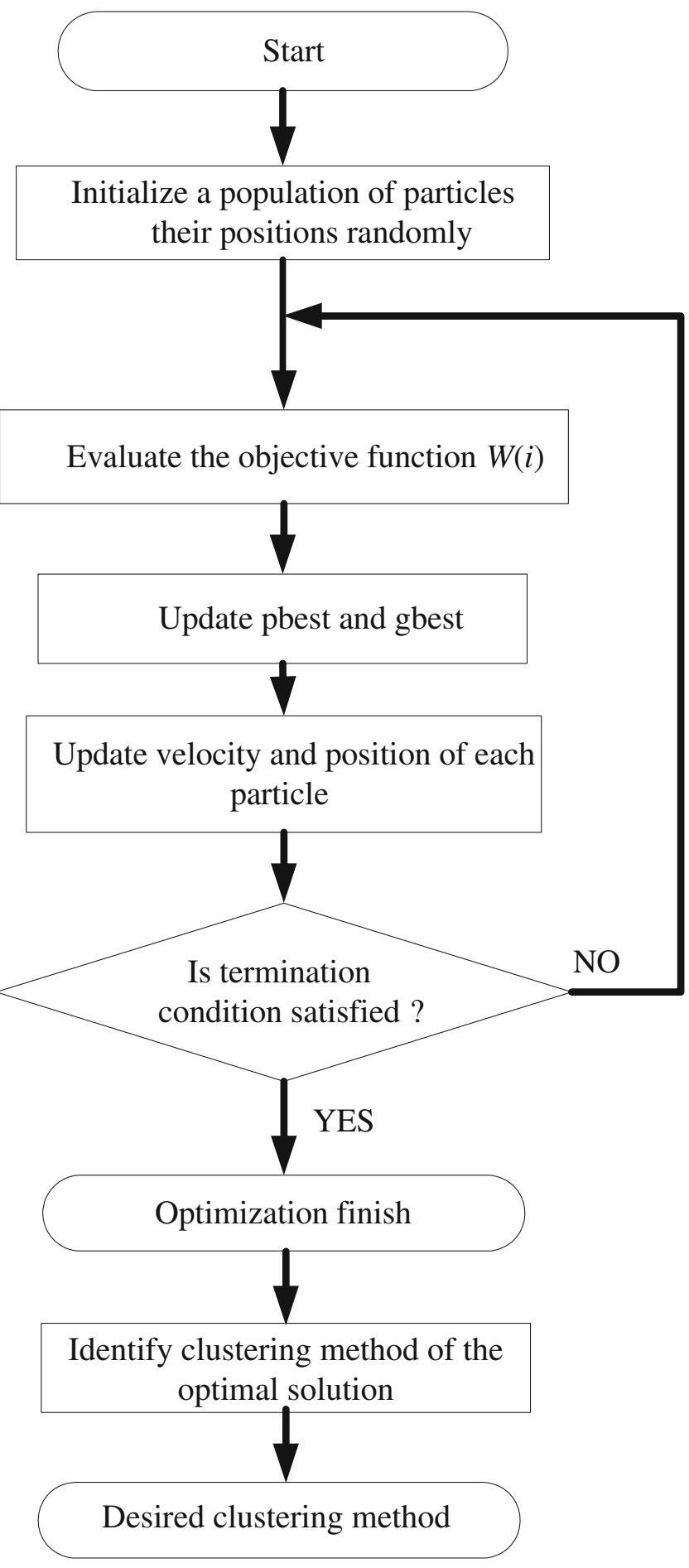

Fig. 3 Flowchart of the algorithm to seek the optimal solution 
a step size. The process of implementing PSO is as follows:

1. Initialize a population array of particles with random positions and velocities on $D$ dimensions in the search space

2. Loop

3. For each particle, evaluate the desired optimization fitness function in $D$ variables

4. Compare particle's fitness evaluation with its $\vec{p}_{i}$. If current value is better than $\vec{p}_{i}$, then set $\vec{p}_{i}$ equal to the current value

5. Identify the entire particle with the best success so far and assign it to the variable $\overrightarrow{p g}$

6. Change the velocity and position of the particle according to the following equations:

$$
\begin{aligned}
\vec{v}_{i}(t+1)= & \omega \overrightarrow{v_{i}}(t)+c_{1} r_{1}\left(\overrightarrow{p_{i}}-\overrightarrow{x_{i}}(t)\right) \\
& +c_{2} r_{2}\left(\overrightarrow{p_{g}}-\overrightarrow{x_{i}}(t)\right) \\
\vec{x}_{i}(t+1)= & \vec{x}_{i}(t)+\vec{v}_{i}(t+1)
\end{aligned}
$$

where $\omega$ is the inertia weighting; $c_{1}$ and $c_{2}$ are acceleration coefficients, positive constraint; $r_{1}$ and $r_{2}$ are the random numbers deferring uniform distribution on [0,1]; and $i$ represents $i$ th iteration

7. If a criterion is satisfied (usually a sufficiently good fitness or a maximum number of iterations), exit loop

8. End loop
Each particle can remember its own previous best value as well as the neighborhood best, and therefore, PSO is more effective than GA in memorizing. PSO also has higher efficiency in maintaining the diversity of the swarm, since all the particles use some information related to the most successful particle in order to improve themselves, whereas in GA, the worse solutions at every generation are discarded and only the good ones are saved for next generation. Therefore, in GA the population evolves around a set of best individuals in every generation. In addition, PSO is easier to implement and there are fewer parameters to adjust compared with GA [29].

\subsubsection{Discrete PSO algorithm description}

The general concepts behind optimization techniques initially developed for problems defined over realvalued vector spaces, such as PSO, can also be applied to discrete valued search spaces where either binary or integer variables have to be arranged into particles [29]. When integer solutions (not necessarily 0 or 1 ) are needed, the optimal solution can be determined by rounding off the real optimum values to the nearest integer. Discrete PSO (DPSO) has been developed specifically for solving discrete problems. DPSO allows discrete steps in velocity and thus in position. In DPSO, the velocity is limited to a certain range $\left[-v_{\max }, v_{\max }\right]$ such that $\overrightarrow{v_{i}}$ always lies in that range. The new velocity and position for each particle $i$ in dimension $d$ is
Fig. 4 Fitness value by the change of iterations for 11 components with population size 5

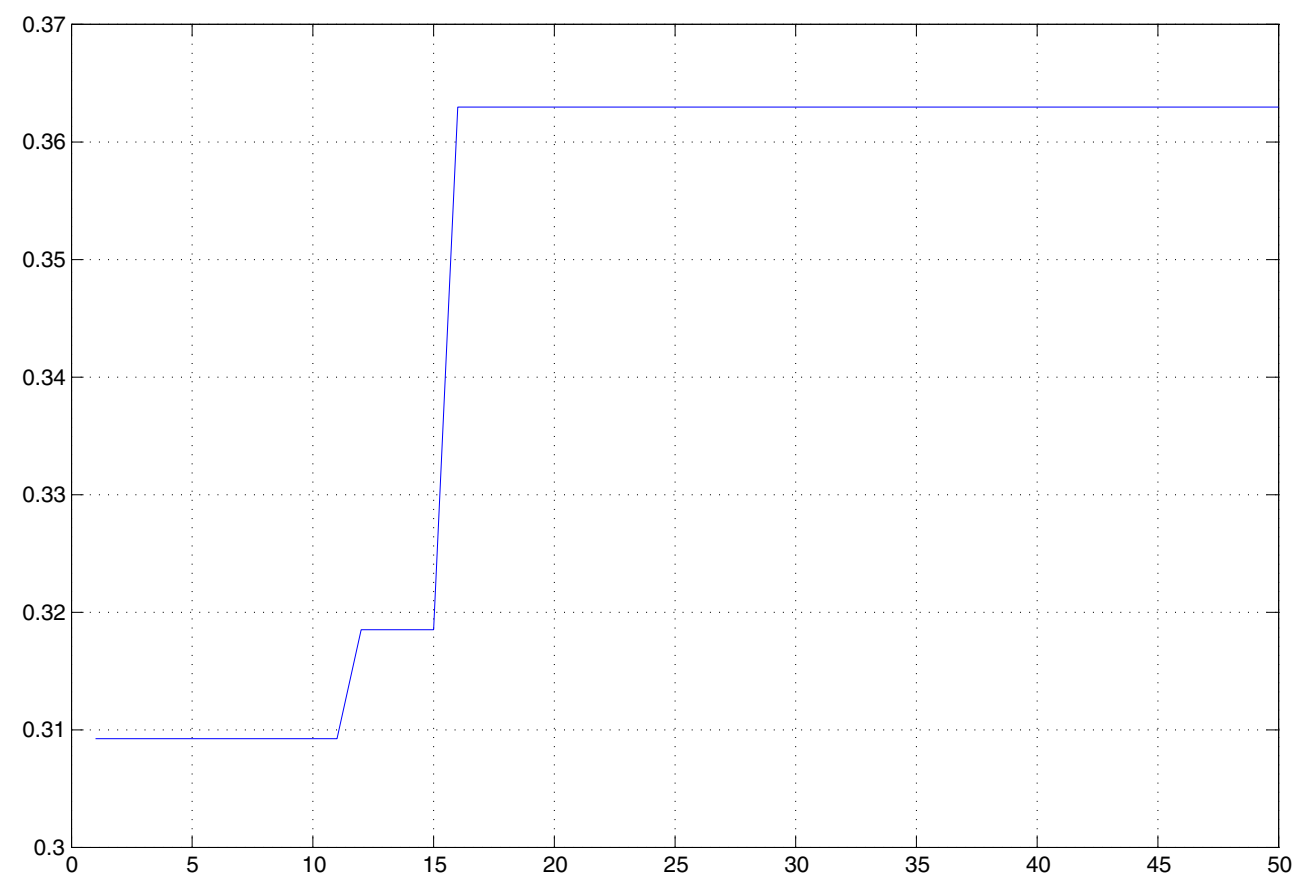




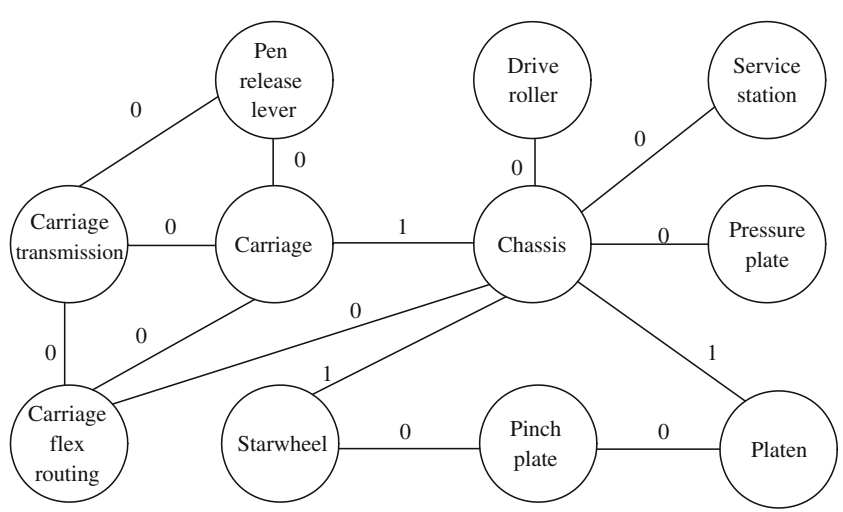

Fig. 5 Values of links corresponding to the optimal solution for the basic structure

determined according to the velocity and position update equations given by Eqs. 4 and 5 .

$$
\begin{aligned}
\vec{v}_{i}(t+1)= & \operatorname{round}\left(\omega \overrightarrow{v_{i}}(t)+c_{1} r_{1}\left(\overrightarrow{p_{i}}-\overrightarrow{x_{i}}(t)\right)\right. \\
& \left.+c_{2} r_{2}\left(\overrightarrow{p_{g}}-\overrightarrow{x_{i}}(t)\right)\right)
\end{aligned}
$$

$\vec{x}_{i}(t+1)=\vec{x}_{i}(t)+\overrightarrow{v_{i}}(t+1)$

In Eq. 4, the value of velocity is binary or integer because round() function can round off the value.

\section{Numerical example}

\subsection{System description}

A mobile printer is introduced in this study to illustrate the calculation procedure of configuration granularity and partition. Figure 2 shows the simplified structure of the printer. Based on Eq. 1, there are 11 components,

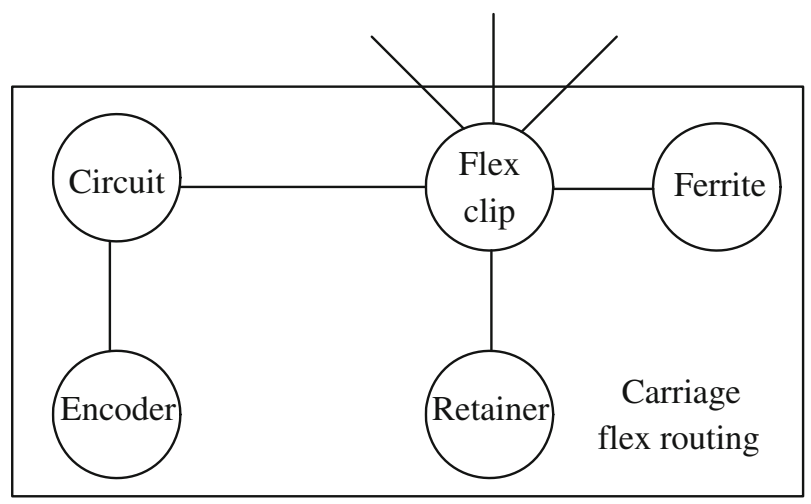

Fig. 6 The decomposition structure of the carriage flex routing
Table 2 Costs of components in the ass-circuit

\begin{tabular}{ll}
\hline Component & Cost $(\$)$ \\
\hline Encoder & 4.5 \\
Circuit & 3 \\
Retainer & 1.2 \\
Ferrite & 1.2 \\
Flex clip & 1.2 \\
\hline
\end{tabular}

and the possible computation scale is from $2^{10}$ to $2^{55}$. The figure gives possible connections among components, and there are 14 connections within the present structure; as a result, the number of clustering methods is $2^{14}$ for this case.

In this example, only cost and diversity are taken as impact factors to simplify the calculation. Therefore, the objective function is:

$$
\begin{aligned}
W_{i} & =\alpha_{1} C(i)-\alpha_{2} N(i) \\
& =\alpha_{1} \frac{C_{\max }-C_{i}}{C_{\max }-C_{\min }}-\alpha_{2} \frac{N_{\max }-N_{i}}{N_{\max }-N_{\min }}
\end{aligned}
$$

where $C_{i}$ denotes the average cost of configuration schemes with the clustering method $i$ and $N_{i}$ means possible diversity numbers in configuration with the clustering method i. $C_{\max }$ and $C_{\min }$ are the biggest value and the smallest value of average costs with all clustering methods, as well as $N_{\max }$ and $N_{\min }$ are two extremes of diversity numbers. $\alpha_{1}$ and $\alpha_{2}$ are positive constants, and their values can be defined by the preferences of customers. In this case, both $\alpha_{1}$ and $\alpha_{2}$ are supposed as 1. Generally, customers prefer cost-effective products and are satisfied with more options. Therefore, for this objective function, it can be concluded that when the

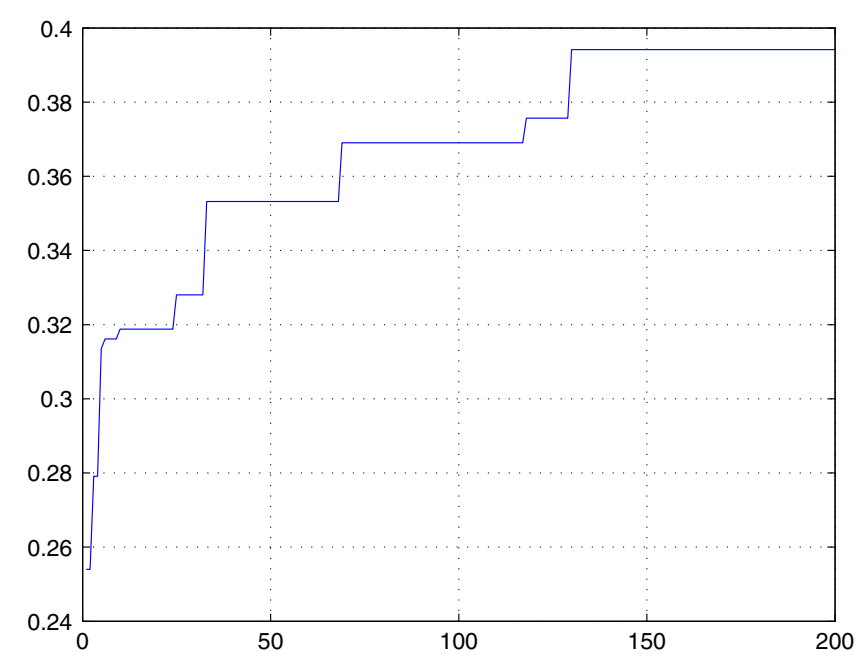

Fig. 7 Fitness value by iterations with decomposed flex routing 
Fig. 8 Values of links corresponding to the optimal solution with decomposed flex routing

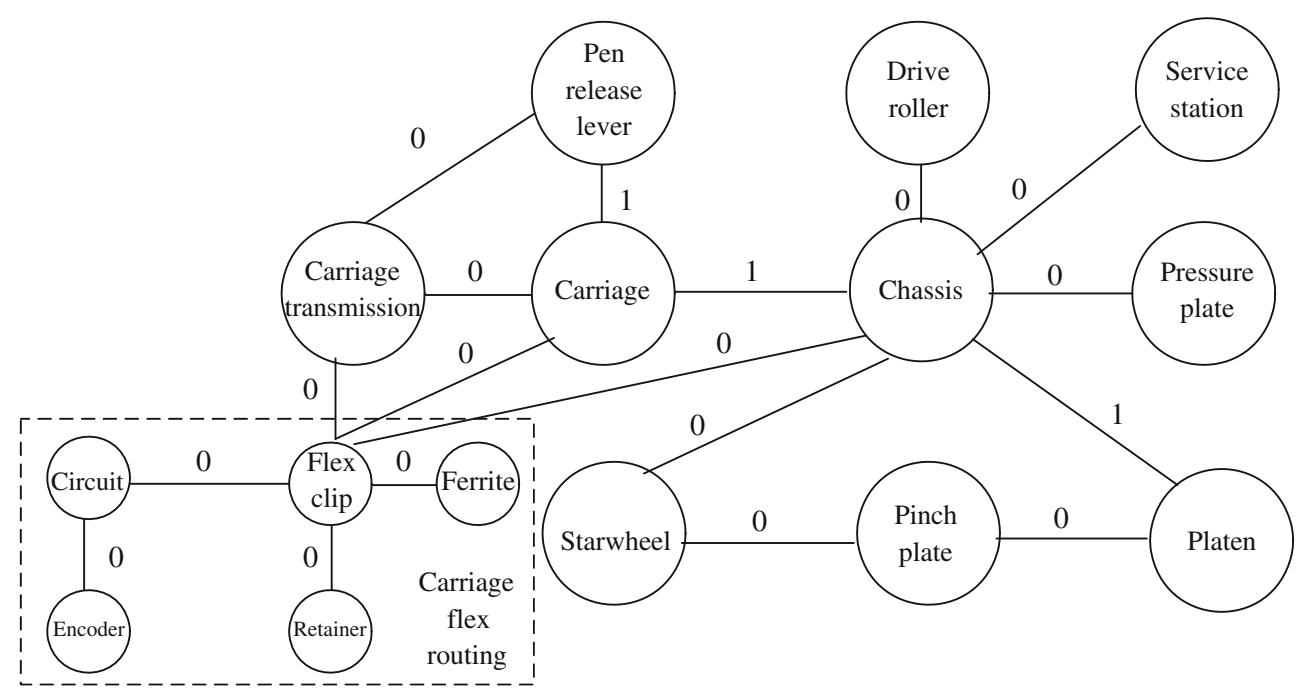

average cost of configuration schemes with a clustering method is less and possible diversity is more, the functional value is bigger and approaches the optimal.

Component costs of the printer are listed in Table 1. In this case, once two components are combined to form a module, the cost of the module is supposed as $80 \%$ of the total cost of these two components. On the other hand, the diversity number of one module is set as 2 , so $N_{i}$ is calculated based on the number of modules $n_{i}$ of a clustering method, $N_{i}=2^{n_{i}}$. For example, if components of the printer are integrated to two modules, the diversity number $N$ is 4 . With more modules in a clustering scheme, the manufacturer can perform better in providing product diversity but need more cost.

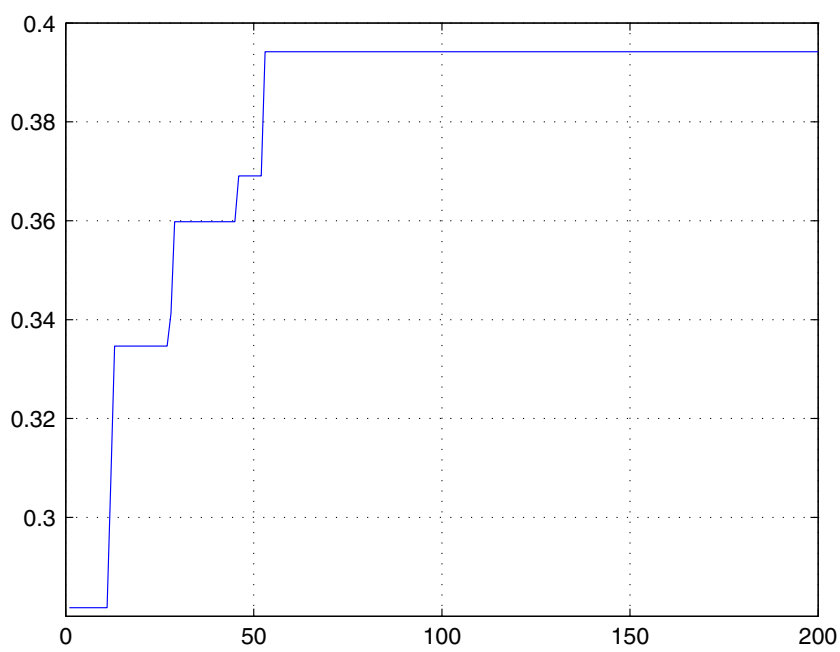

Fig. 9 Fitness value by iterations with population size 15

\subsection{Optimization for the basic structure}

DPSO is introduced here to seek the optimal solution of the objective function. The variable in the algorithm is the set of links among components. When two components connect and are integrated in a module in a clustering method, the link between them is valued as 1 in calculation; otherwise, the link is 0 . The flowchart of the algorithm is shown in Fig. 3.

In optimization, the parameters of DPSO are firstly set as follows: the population size as $5, c 1=c 2=$ $1.5 ; \omega=1.2-0.8$, and total iteration $=50$. Here, the values of $\omega, c 1$, and $c 2$ are defined based on former literatures on PSO. $\omega$ is linearly decreasing. The higher values of $\omega$ are better for a global search, while the lower values suit for a local one. Changing situation

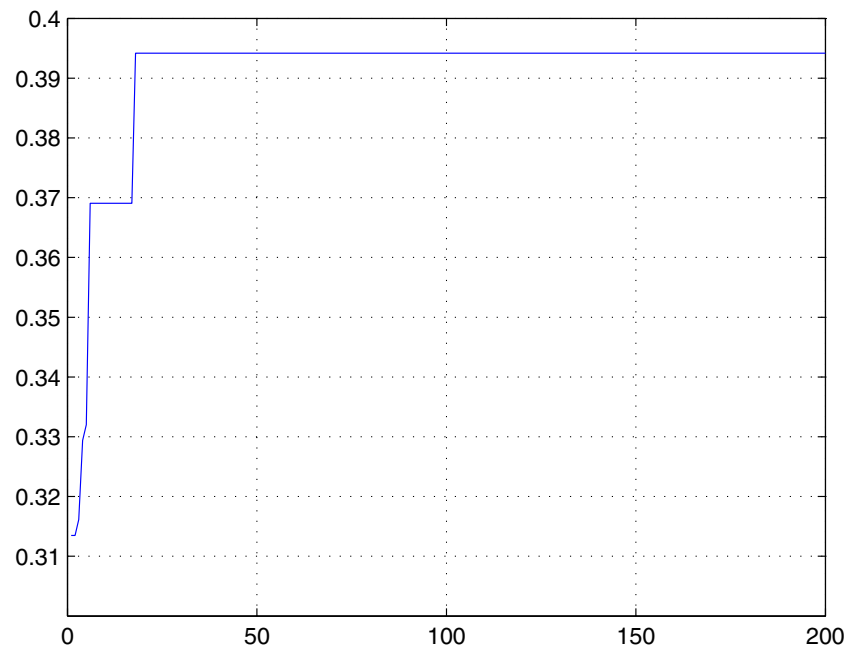

Fig. 10 Fitness value by iterations with population size 30 


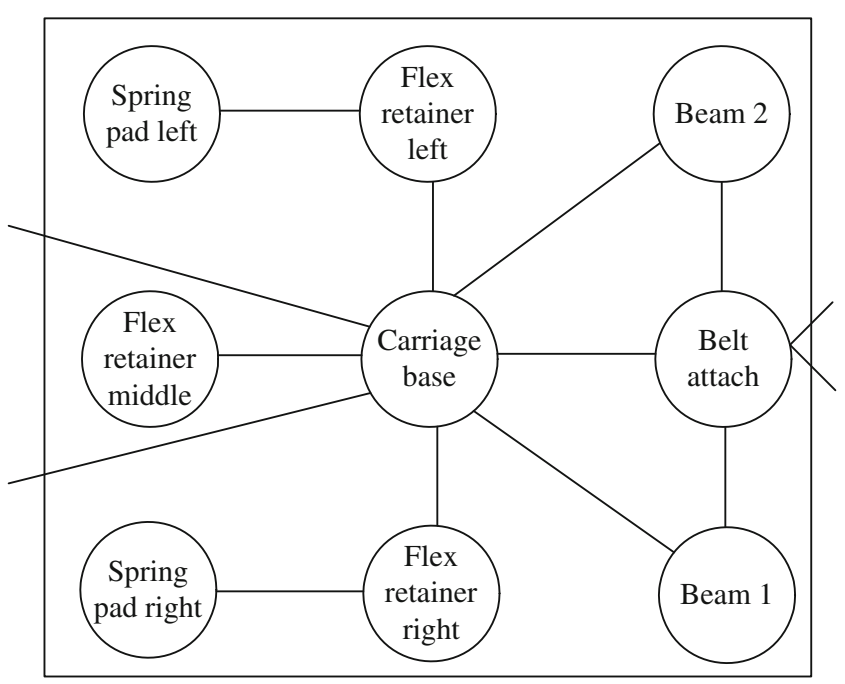

Fig. 11 The decomposition structure of the carriage

of the objective function fitness value with iteration is shown in Fig. 4.

It is not hard to find that the optimal solution can be obtained after 16 iterations. The fitness value is 0.3630 . The authors identify the clustering method corresponding to the optimal solution through a matrix formed by components. Both column and row in the matrix are components involved in configuration, and the elements of the matrix are links among components. From Fig. 5, values of links corresponding to the optimal solution in the algorithm can be found.

According to the values of links, the desired clustering method conforming to the objective function mentioned above is identified: carriage, chassis, starwheel, and platen form a module, and all other components are independent modules, respectively. For such a clustering method, the diversity number in configuration is $2^{8}$.

\subsection{Optimization for the hierarchical structure}

The printer is a hierarchical product to some extent and can be decomposed further. For example, the flex

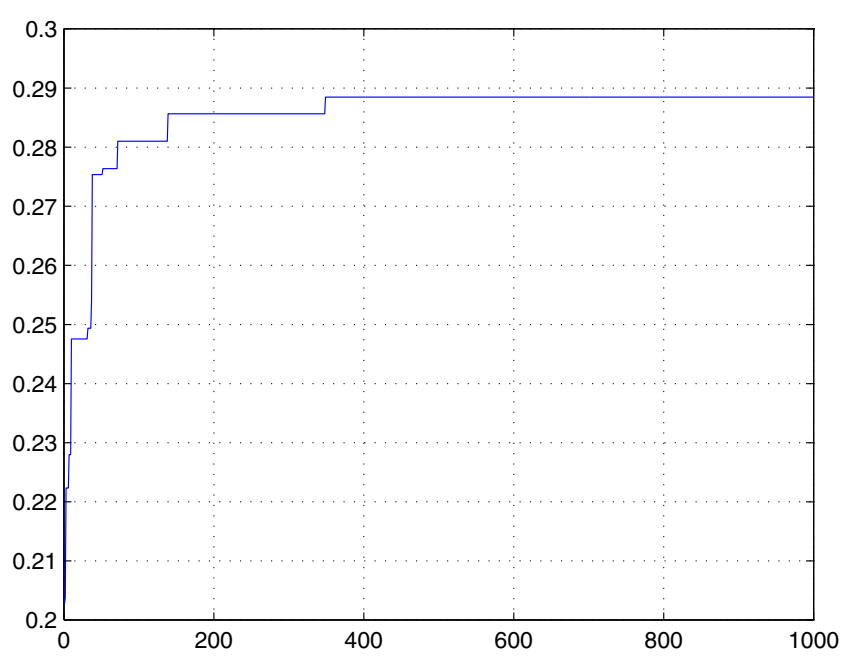

Fig. 13 Fitness value by iterations with 36 links and population size 30

routing is consisted with ass-circuit, retainer, ferrite, and flex clip. Moreover, the ass-circuit includes optical encoder and circuit as shown in Fig. 6, and their individual costs are listed in Table 2. The sum of costs of these components is not equal to the cost of the carriage flex routing in consideration of the cost reduction with integration.

For the structure where the carriage flex routing is decomposed, the computation scale has been enlarged to $2^{18}$ with four new connections. The changing profile of new fitness value by iterations is shown in Fig. 7, where the population size is $5, c 1=c 2=1.5$; $\omega=1.2-$ 0.8 , and total iteration $=200$.

Corresponding values of links to the optimal in Fig. 7 are illustrated in Fig. 8. It can be found that, with the carriage flex routing is decomposed into several smaller components, the optimal clustering method becomes different. The fitness value is 0.3942 . Meanwhile, it noticeable that iterations needed to reach the optimal state increase with a larger computation scale. Here, the authors change the population size of DPSO to 15 and 30 , respectively, and optimization results are shown in
Fig. 12 The decomposition structure of the starwheel

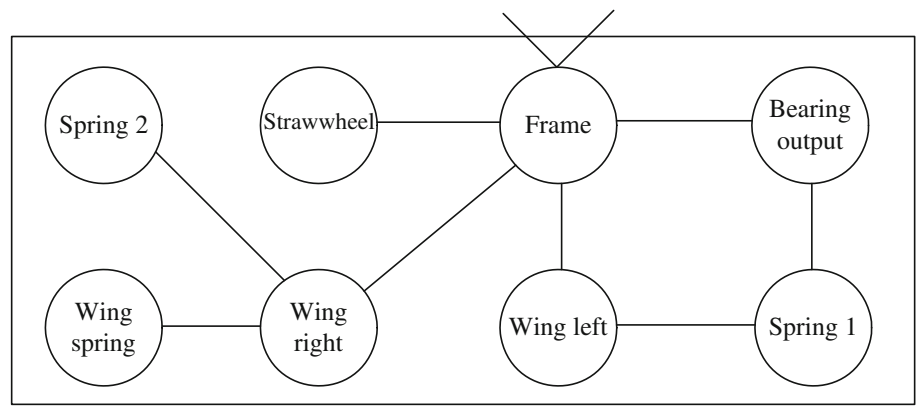


Figs. 9 and 10. Obviously, with a larger population size, the optimal solution can be found with less iterations.

\subsection{Discussions}

To evaluate the adaptability of DPSO to issues of component clustering with larger computation scale, the authors decompose another two components: carriage and starwheel. The internal structures of these two components can be found in Figs. 11 and 12.

With these two decompositions, the computation scale of DPSO has been expanded to $2^{36}$. In the optimization, population size is set as 30 and 50, respectively, and the optimal solution can be found with over 100 iterations as shown in Figs. 13 and 14.

So far, DPSO is effective in optimization of clustering methods for product configuration in the present case. Apparently, with more decomposition and more links, the number of necessary iterations increases. With enlarging the population size in PSO, the optimization can be accelerated. To check the adaptability of DPSO in this kind of issues, the authors generalize the calculation. In details, all components in a product are supposed to be able to connect any of the others, and the population size is fixed as 20 . The authors gradually increase the number of components from 10 to 50 and check whether the optimal is available and how many iterations are needed. In fact, the computation scale of the case with 50 inter-connectable components has been very huge, equal to $2^{1,225}$ theoretically, but Fig. 15 shows that DPSO still can find or at least approach the optimal solution with over 2,000 iterations.

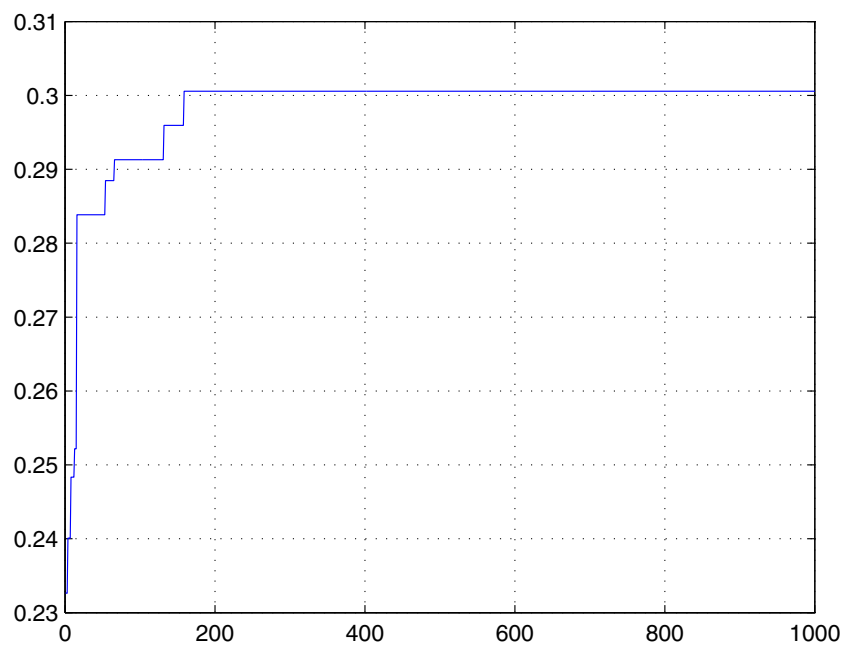

Fig. 14 Fitness value by iterations with 36 links and population size 50

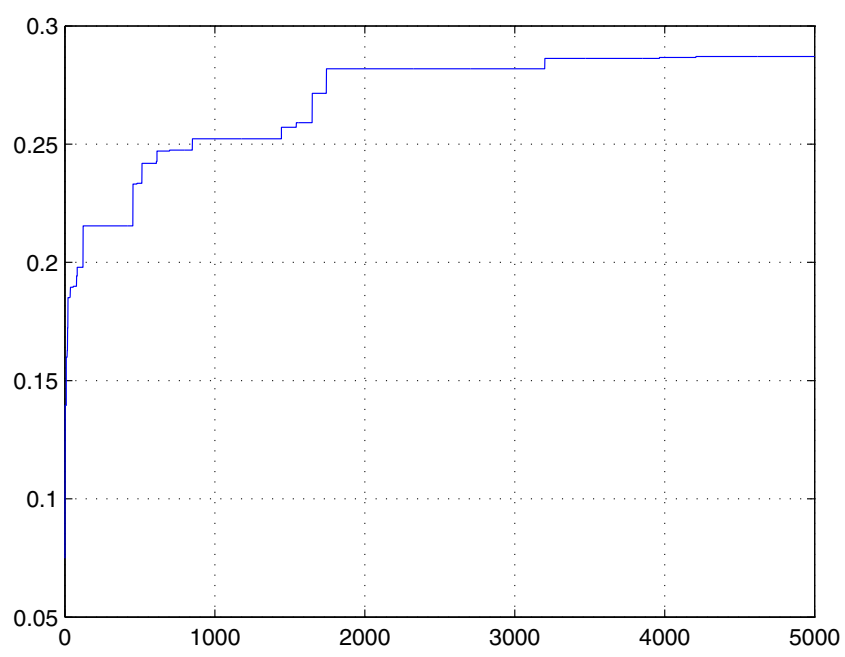

Fig. 15 Fitness value by iterations with 50 inter-connectable components

For possible constraints in connection and structure ignored in former sections, measurements can taken to eliminate their effects before optimization; therefore, the adaptability of the algorithm is not related with those constraints. In particular, the connection matrix and the combination matrix developed in [12] can be applied in configuration to delete incompatible solutions according to coupling strengths between components.

\section{Conclusions and perspectives}

Components in a product can be clustered into several modules according to some requirement. In this paper, the authors define such a clustering issue as an optimization problem and identify its possible computation scale. Discrete PSO is applied to seek the optimal in the whole solution space, and it is proved as an effective method with an example of printer. With the increase of numbers of components in configuration, parameters in the algorithm can be adjusted to speed up the optimization.

For future researches, optimization with other methods, especially with approaches based on artificial intelligence, are helpful for clustering components facing product configuration. Comparison between results in this study and those obtained from other concepts can further evaluate whether PSO is the effective method in this field.

Relative values are used to calculate the fitness of the objective function in the former sections, which limit value of each part in the function into the range of $[0,1]$. Such limitation facilitates the comparison 
between different solutions and simplify the valuing of $\alpha_{i}$. However, based on relatives, there is no internal correlations within fitness values of functions with varying components. If absolute values of objective functions are used in future studies, the relationship of fitness value and computation scale can be explored, and a balance between these two parameters may be helpful to determine the desired partition level for configuration.

In addition, adhesiveness among components, or connectable coefficients between any two components, is neglected in this paper. Sometimes, whether two components can be combined together depends on the coefficients, and these values can be expected to act their roles in comparison and optimization.

Acknowledgements This research is partly supported by National Natural Science Foundation of China (No. 70671071). At the same time, the authors sincerely appreciate for comments of the editor and reviewers.

Open Access This article is distributed under the terms of the Creative Commons Attribution Noncommercial License which permits any noncommercial use, distribution, and reproduction in any medium, provided the original author(s) and source are credited.

\section{References}

1. Pine J II (1993) Mass customization: the new frontier in business competition. Harvard Business School Press, Cambridge

2. Salvador F, Forza C, Rungtusanatham M (2002) Modularity, product variety, production volume, and component sourcing: theorizing beyond generic prescriptions. J Oper Manag 20:549-575. doi:10.1016/S0272-6963(02)00027-X

3. Sabin D, Weigel R (1998) Product configuration frameworks-a survey. IEEE Intell Syst 13(4):42-49. doi:10.1109/5254.708432

4. Jiao R, Tseng M (1999) A methodology of developing product family architecture for mass customization. J Intell Manuf 10:3-20. doi:10.1023/A:1008926428533

5. Chen L, Lin L (2002) Optimization of product configuration design using functional requirements and constraints. Res Eng Des 13:167-182. doi:10.1007/s00163-002-0015-z

6. Ulrich K (1995) The role of product architecture in the manufacturing firm. Res Policy 24:419-440. doi:10.1016/00487333(94)00775-3

7. Krishnan V, Ulrich K (2001) Product development decisions: a review of the literature. Manage Sci 47(1):1-21. doi:10.1287/mnsc.47.1.1.10668

8. Kusiak A, Huang C (1996) Development of modular products. IEEE Trans Compon Packaging Manuf Technol Part A 19(4):523-538. doi:10.1109/95.554934

9. Baldwin C, Clark K (1997) Managing in an age of modularity. Harvard Bus Rev 75(5):84-93

10. Mikkola JH, Gassmann O (2003) Managing modularity of product architectures: toward an integrated theory. IEEE Trans Eng Manage 50(2):204-218. doi:10.1109/TEM. 2003.810826
11. Jiao R, Simpson T, Siddique Z (2007) Product family design and platform-based product development: a state-ofthe-art review. J. Intell Manuf 18(1):5-29. doi:10.1007/s10845007-0003-2

12. Liu Y, Ma Y, Lee S, Thimm G (2007) Product module partition and optimization for customization. Int J Adv Manuf Sys 10(1):9-14

13. Mittal S, Frayman F (1989) Towards a generic model of configuration tasks. In: Proceedings of eleventh International Joint Conference on Artificial Intelligence. San Francisco, pp 1395-1401

14. Aldanondo M, Hamou K, Moynarda G, Lamothe J (2003) Mass customization and configuration: requirement analysis and constraint based modeling propositions. Integr ComputAided Eng 10:177-189

15. Liu Y, Liu Z (2010) Multi-objective product configuration involving new components under uncertainty. J Eng Des 21(4):473-494. doi:10.1080/09544820802474663

16. Brown D (1998) Defining configuring. Artif Intell Eng Des Anal Manuf 12:301-305

17. Forza C, Salvador F 2002. Product configuration and interfirm coordination: an innovative solution from a small manufacturing enterprise. Comput Ind 49(1):37-46. doi:10.1016/ S0166-3615(02)00057-X

18. Viswanathan S, Allada V (2006) Configuration optimization for disassembly planning: a differential approach. Int $\mathrm{J}$ Manag Sci 34:599-616. doi:10.1016/j.omega.2005.01.011

19. Yeh J, Wu T (2005) Solutions for product configuration management: an empirical study. Artif Intell Eng Des Anal Manuf 19:39-47. doi:10.1017/S0890060405050043

20. Yigit A, Ulsoy A, Allahverdi A (2002) Optimizing modular product design for reconfigurable manufacturing. J Intell Manuf 13:309. doi:10.1023/A:1016032714680

21. Xie H, Henderson P, Kernahan M (2005) Modeling and solving engineering product configuration problems by constraint satisfaction. Int J Prod Res 43(20):4455-4469. doi:10.1080/00207540500142381

22. Sosa M, Eppinger S, Rowles C (2007) A network approach to define modularity of components in complex products. J Mech Des 129:1118-1129

23. Andres C, Lozano S (2006) A particle swarm optimization algorithm for part-machine grouping. Robot Comput-Integr Manuf 22(5-6):468-474 doi:10.1016/j.rcim.2005.11.013

24. Duran O, Rodriguez N, Consalter L (2010) Collaborative particle swarm optimization with a data mining technique for manufacturing cell design. Expert Syst Appl 37:1563-1567 doi:10.1016/j.eswa.2009.06.061

25. Sakthivel V, Bhuvaneswari R, Subramanian S (2010) Multiobjective parameter estimation of induction motor using particle swarm optimization. Eng Appl Artif Intell 23:302-312. doi:10.1016/j.engappai.2009.06.004

26. Kennedy J, Eberhart RC (1995) Particle swarm optimization. In: Proceedings of the IEEE international conference on neural networks IV, pp 1942-1948

27. Eberhart R, Kennedy J (1995) A new optimizer using particles swarm theory. In: Proceedings of Sixth International Symposium on Micro Machine and Human Science, pp 3943 doi:10.1109/MHS.1995.494215

28. Eberhart RC, Simpson PK, Dobbins RW (1996) Computational intelligence PC tools. Academic, Boston

29. Del Valle Y, Venayagamoorthy G, Mohagheghi S, Hernandez J, Harley RG (2008) Particle swarm optimization: basic concepts, variants and applications in power system. IEEE Trans Evol Comput 2:178-195. doi:10.1109/TEVC. 2007.896686 